\title{
Decreased CXCL5 expression associated with intrauterine adhesions in a rat model and human endometrial tissues
}

Shiying Sun ( $\sim$ sunsy71@hotmail.com )

The second Affiliated Hospital of Anhui Medical University https://orcid.org/0000-0003-2668-7907

\section{Zi-Ang Fang}

The Second Affiliated Hospital of Anhui Medical University

\section{Yang Gao}

Nanyang Technological University

\section{Yu He}

The Second Affiliated Hospital of Anhui Medical University

\section{Chao Sun}

The Second Affiliated Hospital of Anhui Medical University

\section{Lei Zhan}

The Second Affiliated Hospital of Anhui Medical University

\section{Guiju Zhou}

The Second Affiliated Hospital of Anhui Medical University

\section{Bing Wei}

The Second Affiliated Hospital of Anhui Medical University

\section{Research article}

Keywords: CXCL5, Intrauterine adhesion, Asherman syndrome, IUA rats

Posted Date: May 27th, 2020

DOI: https://doi.org/10.21203/rs.3.rs-23687/v1

License: (1) (1) This work is licensed under a Creative Commons Attribution 4.0 International License. Read Full License 


\section{Abstract \\ Background}

Intrauterine adhesion (IUA) is a consequence of endometrium damage, leading to infertility, recurrent miscarriage, and various complications of pregnancy. IUA usually occurs after an infection or injuryrelated inflammation of the endometrium. CXCL5, a member of chemokines, is associated with the remodeling of connective tissues, but its roles in the formation of IUA remain uncertain. This study aims to investigate the expression and effect of CXCL5 in the development of IUA.

\section{Results}

CXCL5 protein level in the endometrium of IUA rat is significantly decreased compared with that in normal and sham-operated endometria of rats $(P<0.001)$. Furthermore, real-time PCR and western blotting assays also show that the CXCL5 expression in endometrial tissues from IUA patients is much lower than that in normal endometrium $(P<0.01)$, which is consistent with the results based on the rat model. CXCL5 significantly up-regulates MMP9 expression and slightly up-regulates p65 expression in human endometrial cells after CXCL5 overexpression.

\section{Conclusions}

These results show that CXCL5 plays an important role in the inhibition of IUA formation after injury by modulating MMP9 expression and therefore has a protective effect of CXCL5 in the adhesion formation of IUA. These findings provide valuable information for evaluating prognosis and guiding therapy of intrauterine adhesions.

\section{Background}

Intrauterine adhesion (IUA), also known as Asherman syndrome, is characterized by adhesions and fibrosis in endometrium within the uterus and/or cervix, and partial or complete obliteration of the uterine cavity [1, 2]P. IUA occurs most commonly and frequently after trauma or infection, such as a simple operation on the uterus like a cesarean section, especially during the postpartum period with low estradiol levels [3]. The most common early symptoms of IUA patients are menstrual abnormalities, recurrent abdominal pain, and amenorrhea [4]. IUA often leads to infertility, recurrent miscarriage, and various complications of pregnancy, such as placenta implantation, placental abruption, preterm premature rupture of membranes, and developmental abnormalities in the fetus as a result of the loss of normal endometrium and insufficient the blood supply to the uterus [5-7]. In recent years, IUA has become one of the common causes of female infertility in China, and its incidence has been on the rise with uterine cavity surgery increasing $[4,8]$. Thus, the prevention and treatment of IUA are of great value in maintaining women's health as well as meeting fertility requirements. 
Very recently, hysteroscopy has been widely considered a gold standard to both diagnose and treat IUA. Meanwhile, various techniques for adhesiolysis and prevention of scar formation have also been applied in the clinic [9]. Indeed, the hysteroscopy with lysis of the scar tissue shows its huge advantage of restoring normal uterine function in many cases. However, such therapy is inapplicable in severe cases due to the likelihood of recurrence after surgical repair [10]. Reported statistics show that the postoperative endometrial recovery and clinical cure rate of patients with severe IUA are much worse than those with mild and middle IUA $[11,12]$. These results indicate that the clinical prognosis of IUA patients is related to the degree, range, residual endometrial area before surgery, and endometrium growth. In order to further reveal the progression and relative molecular mechanisms underlying IUA, molecular biology studies are highly desired for the next-generation treatment and prevention of the postoperative readhesion of patients.

The basal layer is an essential structure in the human endometrium, located between the myometrium and the functional layer [6]. It contains various progenitors and stem cells that provide the regenerative capacity and participate in the remodeling of the endometrium after the loss of the functional layer in physiological menstrual cycles $[9,13]$. Once the basal layer of the endometrium is severely damaged, endogenous endometrial stem cells are damaged, and thus endometrial regeneration ability is lost, usually leading to IUA $[14,15]$. More importantly, a broad range of inflammatory chemokines, together with cytokines and cell adhesion molecules, are abnormally expressed during the development of IUA and participate in the regulation of secretion and the degradation of extracellular matrix components in the fibrosis of the endometrium $[15,16]$. Therefore, the urgency for exploring the mechanism underlying the role of inflammatory chemokines in the process of adhesion formation has been raised for providing new therapeutic targets for IUA.

CXC motif chemokine ligand 5 (CXCL5), also known as epithelial neutrophil-activating peptide-78 (ENA78), is a member of the CXC subfamily of chemokines and first cloned in 1994 by Chang [17]. After binding with its receptor CXCR2, CXCL5 enables the recruitment of neutrophils followed by the promotion of angiogenesis and the remodeling of connective tissues [18]. Recently, Konduru, A.S. reported that the knockdown of endogenous p65 suppresses CXCL5 gene transcription in vivo [19], indicating that the CXCL5 expression level is regulated by the NF-KB pathway. In contrast, the silence of CXCL5 expression inhibited the NF-KB pathway in cells also has been demonstrated [20]. Moreover, the mRNA and protein levels of p65 significantly increase and associate with the degree of adhesion in IUA patients [21, 22], suggesting CXCL5 might be involved in the development of IUA mediated by the NF-KB pathway. It is also noted that MMP9, a class of the matrix metalloproteinase, plays an essential role in the degradation of the extracellular matrix and the fibrous tissue of IUA patients. The low expression of MMP9 in IUA patients results in excessive deposition of extracellular matrix as well as the normal endometrium is replaced by fibrous connective tissue, ultimately leading to endometrium fibrosis and adhesion formation of the endometrium [23]. Although the CXCL5/CXCR2 axis is essential for the cell adhesion and migration followed by matrix remodeling by activating PI3K/AKT-induced up-regulation of MMP9 in bladder cancer [24], the role of CXCL5 in the process of the adhesion formation and fibrosis proliferation in IUA remains unclear. 
In this study, we investigate the expression of CXCL5 and its potential role in IUA. CXCL5 has a decreased expression both in IUA animal models and patient endometria. Such low expression of CXCL5 may induce MMP9 decreased expression and lead to the formation of intrauterine adhesion. These results show that CXCL5 plays a key role in inhibiting IUA formation and thus is a promising therapeutic target for the prevention and treatment of IUA.

\section{Results}

\section{Morphological changes of endometrium in IUA animal models}

Previous studies have shown a correlation between the chemokines with the degradation of the extracellular matrix and fibrosis $[25,26]$. In order to investigate the potential role of CXCL5 in IUA pathogenesis, we first established a rat model of endometrium adhesion with phenol mucilage. All rat uteruses were resected after 14 days of operation, and the gross morphology displayed that contracture occurred in rats' uterus of the IUA group rather than that in normal and sham-operated groups (Fig. 1A-C). Microscopy images in Fig. 1D-F show that the endometrial cavity surface is covered by regularly arranged columnar epithelium with abundant endometrial glands both in control and sham-operated groups. Compared with the normal and sham-operated groups in Fig. 1, the uterine endometrial glands of IUA rat models are remarkably reduced with tissue hyperplasia, and large amounts of macrophages and plasma cells are replaced by collagen fibers, indicating that inflammation is involved in IUA pathogenesis.

\section{Degree of endometrial fibrosis in the IUA rat model}

To evaluate the degree of endometrial fibrosis, we performed Masson staining and observed a lot of stromal blue collagen fibers in the IUA model group, which were tightly arranged in the endometrium (Fig. 2). Besides, the histological examination of IUA uterus shows the presence of endometrium adhesion and fibrosis. HE and Masson staining results confirm the IUA rat model was successfully established.

\section{CXCL5 down-regulation associated with IUA in rat models}

Previous studies $[20,21,23,24]$ imply that the abnormal expression of CXCL5 might play an important role in the occurrence of IUA according to existing publications. To this end, we examined the CXCL5 expression in the endometrium of the IUA rats, sham-operated, and normal group by immunohistochemistry. The uteruses of normal and sham-operated groups show intense positive CXCL5 staining, which is mainly expressed in the cytoplasm of glandular epithelial and stromal cells (Fig. 3). The staining quantification indicates that CXCL5 expression level in the IUA group is significantly lower than those in both the sham-operated group and normal group, while there is no obvious difference between normal and sham-operated groups (Fig. $3 \mathrm{~J}$ ). All these results indicate that CXCL5 is involved in the suppression of the endometrium adhesion formation during the genesis and development of IUA. 


\section{Reduced expression of CXCL5 in endometria of IUA patients}

In order to further confirm the low expression of CXCL5 in IUA patients, we then examined the expression level of CXCL5 in the endometria of 6 IUA patients and 6 normal patients, respectively. The mRNA expression level of CXCL5 in the endometrial samples of IUA patients (Fig. 4A) is significantly downregulated $(P<0.01)$ compared with the normal endometria (Ctrl). Similarly, CXCL5 protein expression in the endometria of IUA patients also is remarkably lower than that in the normal endometria (Fig. 4B). Figure 4C shows that the statistically significant difference of CXCL5 level $(P<0.01)$ during the two groups of patients. These results indicate that a reduced expression level of CXCL5 exists in endometrial tissues both of IUA rats and patients, and give strong evidence that CXCL5 plays a protective role in inhibiting genesis of IUA.

\section{CXCL5 positively regulates MMP9 and p65 expression in endometrial cells}

It has been reported that low expression of MMP9 exists in IUA endometrial tissues, and its protein level can be regulated by CXCL5 through PI3K/AKT signaling [23]. Recently, Xue et al. demonstrated that the p65 expression is increased in IUA, and CXCL5 and p65 positively promote the protein expression of each other [22]. To further confirm the correlation between the expression of CXCL5, MMP9, and p65 in IUA endometrial tissues, we performed western blotting to detect the protein level of CXCL5, MMP9, and p65 after transfecting overexpression plasmid of CXCL5 in Ishikawa cells. A significant increase in MMP9 expression and a slight increase in p65 expression were observed with the increase of CXCL5. Representative results are shown in Fig. 4D, and the relative expression of proteins was calculated in Fig. 4E, indicating that the overexpressed CXCL5 up-regulates both MMP9 and p65 protein levels in human endometrial cells. It is important to point out that the low expression of CXCL5 in the endometrium of both IUA rats and patients in Figs. 3 and 4, and previous studies have shown that MMP9 is poorly expressed while p65 is highly expressed in IUA samples [22, 27]. These facts confirm that the low expression of CXCL5 inhibits the adhesion formation in IUA by decreasing the protein level of MMP9 rather than $\mathrm{p} 65$.

\section{Discussion}

IUA is the most common syndrome affecting fertility and pregnancy outcomes and usually occurs after an infection or injury-related inflammation [3]. The present clinical treatment of IUA is mainly restoring the shape of the uterine cavity, but the efficacy is unsatisfactory in the repair of endometrium function [9], driving the enthusiasm for research on the molecular mechanism of IUA pathogenesis. Here, we first demonstrate a reduced CXCL5 expression in the endometrium of both IUA rats and patients and show that CXCL5 plays key roles in protecting endometrium and preventing fibrosis and adhesion formation. 
Collecting a large number of human endometrium samples is known to be difficult in obtaining ethical approval from the Ethics Committee, together with the experimental time and cost. Compared with clinical samples, the IUA rat model is more widely used for the immunohistochemistry analysis of endometrium in IUA $[6,28]$, because it can closely mimic the development and pathological characteristics of IUA patients and its experimental conditions can be easy to control. To investigate the molecular mechanism of IUA genesis, we established an IUA rat model to mimic the condition in human IUA by using the phenol mucilage method. Different from many other model animals, A rat has two distinct cervices and uterine bodies, which is an important advantage for studying IUA. The pathological characteristics of rats more closely mimic the human disease, and the cost in feeding rats is very low, compared with other larger animals such as rabbits. On the other hand, the size of the rat is still large enough for the convenience of performing surgical procedures compared with other smaller animals such as mice. Therefore, rats are commonly used for establishing an ideal model for uterine diseases, particularly for IUA.

Phenol mucilage is widely used for establishing the inflammation-induced adhesion animal model in IUA $[21,29,30]$ and other various medical studies, such as peritoneal adhesion [31], cervicitis [32], pelvic inflammatory [33], et al. for its high effectivity and reliability. Phenol mucilage contains 3 toxic ingredients, including liquefied phenol, Arabic gum, and glycerin. Liquefied phenol is a chemical burn agent, which is highly corrosive and can destroy local tissues and cause an inflammatory reaction. Arabic gum and glycerin are thickeners, which can reduce the damage to adjacent tissues due to the diffusion of phenol. This chemical method is more controllable and easier to operate than the mechanical injury method, and therefore is suitable to be used as a relatively steady and reproducible inflammation-induced animal model of IUA. Although inflammation at the site of endometrial injury is widely considered as the leading cause of IUA [1-3], other factors such as mechanical damage may also lead to IUA and even have been used to make IUA model in animals [28]. To this end, it is necessary to establish a shamoperated group to exclude mechanical damage, including the effects of surgical procedures and injection process on the IUA model rats and thus to confirm that the pathological characteristics in IUA group are only caused by a given concentration of toxic agents. In order to simulate the endometrial injury of IUA rats caused by the operation and injection, we established a sham-operated group by the treatment with PBS, which is the non-toxic agent $[28,30]$. This IUA model is not only beneficial for the understanding of IUA phenotype, molecular mechanisms of adhesion formation, but is also useful for choosing the effective therapeutic strategies for IUA prevention and treatment. As mentioned above, the stricture appearance of the uterus at the IUA model rats was observed, endometrium becoming thin and atrophic, the number of glands reduced, and collagen fibers proliferated significantly (Figs. 1 and 2). These changes are consistent with the pathological manifestation of human IUA, confirming that our model is a qualified IUA rat model.

The immunohistochemistry results of rats show that the protein level of CXCL5 in the IUA group is significantly lower than those in the normal and sham-operated groups (Fig. 3). Additional measurements of mRNA level and protein level of CXCL5 in humans also confirm the low expression of CXCL5 in the endometria of IUA patients (Fig. 4A-B). The above results suggest that the expression of CXCL5 is 
associated with the maintenance of normal intimal functions and should play an important role in inhibiting IUA formation.

Previous studies show that CXCL5 is involved in PI3K/AKT signal pathway to regulate the expression of matrix metalloproteinases (MMPs), which play critical roles in fibrogenesis and fibrosis [24]. After binding to its receptor CXCR2, CXCL5 induces the elevated phosphorylation of AKT and activates PI3K/AKT signaling pathway. MMP9, a group of hydrolases, can degrade type IV and $\mathrm{V}$ collagens and is essential for the breakdown of extracellular matrix in normal physiological and disease processes [34]. In addition, Hu et al. demonstrated the low MMP9 expression in endometrial tissues of IUA and indicated the lack of MMP9 expression is closely related to the IUA development [27]. Combined with the above important fact, the significantly reduced expression of CXCL5 in both IUA animal models and patient endometria found in this study indicates that the low CXCL5 expression leads to the reduced expression of MMP9. This conclusion, together with our second finding that the enhanced CXCL5 expression in endometrial cells leads to the increased expression of MMP9, reveals an evidently positive correlation between the expressions of CXCL5 and MMP9. Based on the above analyses, we suggest a molecular mechanism of CXCL5 in IUA as follows: the low expression of CXCL5 induces to a decreased expression of MMP9 through the PI3K/AKT signaling pathway, leading to the accumulation of extracellular matrix and proliferation of collagen fibers on the damaged endometrium; then normal endometrium growth is inhibited; finally, intrauterine adhesion is formed.

CXCL5 can up-regulate the p65 expression and activates the NF-KB signaling pathway, while p65 can also bind the promoter of the CXCL5 gene and induces its transcription in cancer cells $[19,35]$. Very recently, evidence accumulated shows that the NF-KB pathway is associated with disorders of fibrogenesis and up-regulated in IUA genesis [21]. However, it remains unclear whether CXCL5 regulates the p65 expression in the NF-KB signaling pathway in IUA. Importantly, Xue et al. found the overexpression of p65 in IUA [22]. To further investigate the correlation between p 65 and the low-expressed CXCL5 in IUA found in this study, we detected the p65 expression level in CXCL5-overexpressed endometrial cells. Surprisingly, we observed a slight increase in p65 protein level with the increase of CXCL5 expression in endometrial cells, indicating that the p65 expression is not merely regulated by CXCL5 in IUA, and its increased expression in IUA can be attributed to the regulation by other more important factors. Thus, we speculate that the NF$\mathrm{KB}$ signaling pathway is not the principle molecular mechanism by which CXCL5 inhibits the formation of adhesion in IUA.

\section{Conclusions}

CXCL5 shows a significantly reduced expression in endometrial tissue of IUA both in rats and humans, and CXCL5 positively regulates MMP9 expression, indicating that CXCL5 plays a protecting role in the adhesion formation and fibrogenesis of IUA through modulating MMP9 and therefore is a potential biomarker. Based on these findings, we proposed a molecular mechanism of CXCL5 in IUA. Our results provide important information for evaluating and target the therapy of intrauterine adhesions. 


\section{Methods}

\section{Patients and tissue samples}

Intrauterine adhesion and control tissues were collected from The Second Affiliated Hospital of Anhui Medical University between October 2017 and July 2018. 6 endometrial tissues were collected from intrauterine adhesion patients diagnosed by hysteroscopy, and 6 normal endometrium tissues from secondary infertility control individuals were collected as controls. There is no significant difference in age, weight, or parity between the intrauterine adhesion and control group. The study was approved by the Ethics Committee of the Anhui Medical University (No. 20160034) and complied with the Declaration of Helsinki. All patients of this study were aware and signed an informed consent agreement.

\section{Creation of a rat model for intrauterine adhesions}

Adult female Sprague-Dawley (SD) rats aged 8 weeks (weight: $220 \mathrm{~g}$ ) were obtained from the Experimental Animal Center of Anhui Medical University and used to create the IUA model by phenol mucilage as the description [21,32]. All rats were raised in the laboratory for 1 week of adaption. Vaginal smear was used to evaluate the phases of the estrous cycle, and all animal surgeries were performed during their diestrus stage of the cycle. 40 rats were randomly grouped into three groups: IUA group (20 rats for phenol mucilage treatment), sham-operated group (10 rats with vehicle treatment), and normal group (10 rats with no treatment). Firstly, 5\% pentobarbital sodium was intraperitoneally injected for the anesthetization of rats. Then, intrauterine injections of $0.04 \mathrm{ml}$ phenol mucilage (Liquefied phenol $25 \%$ $\mathrm{v} / \mathrm{v}$, Arabian gum $5 \% \mathrm{w} / \mathrm{v}$, Glycerin $20 \% \mathrm{v} / \mathrm{v}$ ) were administered on adult female rats to cause endometrial injury in IUA group. The sham-operated group received an equal volume of PBS. The rats were housed with free access to food and water in an environment-controlled room at $22^{\circ} \mathrm{C}$ with a $12 \mathrm{~h}$ light and dark cycle. Two weeks after the surgery, all rats were euthanized using a $\mathrm{CO}_{2}$ flow at a rate of $10 \mathrm{~L} / \mathrm{min}$ for 7 min, and endometrium tissue samples were collected for further analyses. This study was performed in strict accordance with the recommendation from the Guide for the Care and Use of Laboratory Animals of the National Institutes of Health. All animal experiments were performed at the Anhui Medical University and approved by the Ethics Committee on Animal Research of the Anhui Medical University (No. LLSC20180106).

\section{H\&E staining and Masson staining}

Endometrium tissues underwent standard paraffin embedding and were cut into 5- $\mu \mathrm{m}$ thick serial sections [36]. Hematoxylin \& eosin (H\&E) staining and Masson staining were performed as previously described. For HE staining, the sections were incubated with hematoxylin solution for 5 min and then stained with eosin solution for 3 min. For Masson staining, after deparaffinization and rehydration, the 
sections were stained with hematoxylin for 5-10 min, and Masson staining mixture for $5 \mathrm{~min}$. Then the sections were immersed in Phosphomolybdic Acid Solution for $1 \mathrm{~min}$ and Aniline Blue Solution for $5 \mathrm{~min}$ and then examined and photographed by microscopy.

\section{Immunohistochemistry and scoring}

Immunohistochemistry was performed according to the manufacturer's instructions (Beijing Zhongshan Golden Bridge). Fully processed with $3 \% \mathrm{H}_{2} \mathrm{O}_{2}$ for 15 min and washed by PBS (phosphate-buffered saline) buffer, then the sections were blocked by $10 \%$ goat serum for $30 \mathrm{~min}$ at room temperature. Antibody against CXCL5 (ab198505, Abcam Company) was incubated with the sections overnight at $4{ }^{\circ} \mathrm{C}$. After incubated with biotinylated secondary antibody and biotinylated horseradish peroxidase complex for $15 \mathrm{~min}$, the sections were visualized by diaminobenzidine (DAB).

The number of CXCL5 positive cells was counted in three randomly chosen fields using a semiquantitative method by multiplying the multiplication of the number of positively-expressed cells and their positive intensity [21]. The proportion of positive cells classified into 5 grades (0-4): $0 \%, 25 \%, 50 \%$, $75 \%$ and $100 \%$. The intensity of the stain was scored from 0 to 3 points: 0 -no staining, 1-weak, 2moderate, 3-strong. The score was calculated by multiplying the proportion and intensity score (0-12). The samples were observed under a light microscope (40x, 100x, 200x, 400x magnification).

\section{RNA isolation and real-time PCR analysis}

Total RNA from the endometrium tissues was isolated, as previously described [37]. RNA was extracted using RNAiso Plus reagent (TaKaRa Biotechnology). RNA samples $(2 \mu \mathrm{g})$ were further reverse-transcribed with the PrimeScriptPTMP RT Master Mix (TaKaRa Biotechnology) according to the manufacturer's instructions. The PCR amplification was performed by using SYBR ${ }^{8}$ Premix Ex TaqP ${ }^{T M} P$ II (TaKaRa Biotechnology). CXCL5 primer sequence is: 5'-CTCAAGCTGCTCCTTTCTCG-3', 5'GCGATCATTTTGGGGTTAAT-3'. GAPDH primer sequence is: 5'-GAAGGTGAAGGTCGGACTC-3', 5'GAAGATGGTGATGGGATTTC-3'. All PCR reactions were run in a multiplex real-time PCR machine (StepOne, Applied Biosystems, USA). GAPDH quantification served as an internal control for normalization. Relative differences in mRNA levels over control values were calculated using the $\triangle \mathrm{Ct}$ method according to the manufacturer's protocol. The results of PCR reactions were independently repeated at least twice.

\section{Cell culture and transfection}


Ishikawa cells, a human endometrial epithelial cell line, were obtained from the American Type Culture Collection (ATCC). Cells were cultured in RPMI-1640 medium (Invitrogen) supplement with $10 \%$ fetal bovine serum (Invitrogen) and antibiotics under the condition of $37^{\circ} \mathrm{C}$ and $5 \% \mathrm{CO}_{2}$. CXCL5 expression vector $(2 \mu \mathrm{g}, 1 \mu \mathrm{g})$ and control plasmid pcDNA3.1 were transfected to cells using jetPRIME transfection reagent (Polyplus) according to the description of the protocol. After incubation with the transfection mix for $24 \mathrm{~h}$, then the cell growth medium was replaced, and the cells were harvested from the dishes following another $24 \mathrm{~h}$ incubation. Finally, the cells were assayed by Western blotting.

\section{Western Blotting}

Cells and tissue samples were lysed with a RIPA buffer containing a protease inhibitor cocktail (B14001, Bimake). Protein concentrations were measured by bicinchoninic acid assay (BCA). Equal amounts of protein mixtures $(30 \mu \mathrm{g})$ were run on SDS-PAGE; the proteins were transferred to PVDF membrane (Millipore) and incubated in PBST (Tween-20) with $5 \%$ non-fat milk for $1 \mathrm{~h}$ at room temperature. The blot was incubated with primary antibodies including anti-CXCL5 (1:100, no. ab198505, Abcam Company), anti-MMP9 (1:1000, no. 3852, Cell Signaling Technology), anti-p65 (1:1000, no. 8242, Cell Signaling Technology) and anti- $\beta$-actin (1:20000, no. KC-5A08, Shanghai Kangcheng) overnight at $4^{\circ} \mathrm{C}$. Secondary antibodies that were conjugated to horseradish peroxidase were incubated with the blot for $1 \mathrm{~h}$ at room temperature. The proteins that were revealed by Western blotting were visualized by using the enhanced chemiluminescence reagents (ECL, Servicebio). The densities of bands were analyzed by ImageJ software and normalized to GAPDH.

\section{Statistical Analysis}

All the data are expressed as mean \pm standard deviation (SD) and analyzed by GraphPad Prism software version 6.0 (San Diego, CA, USA). Comparisons between groups were studied by Student's $t$-test. For analysis of immunohistochemistry staining, a two-way ANOVA test was used. $P<0.05$ was considered statistically significant.

\section{Abbreviations}

CXCL5, CXC motif chemokine ligand 5; CXCR2, CXC motif chemokine receptor 2; DAB, diaminobenzidine; ENA-78, epithelial neutrophil-activating peptide-78; GAPDH, glyceraldehyde-3-phosphate dehydrogenase; $\mathrm{HE}$, Hematoxylin and eosin; IUA, Intrauterine adhesions; MMP9, matrix metalloproteinase 9; MMPs, matrix metalloproteinases; NF-кB, nuclear factor kappa-B; PBS, Phosphate Buffered Saline; PBST, Phosphate Buffered Saline Tween 20; PVDF, Polyvinylidene Fluoride.

\section{Declarations}


Ethics approval and consent to participate

This study was approved by the Ethics Committee of the Anhui Medical University, China (No. 20160034). Written informed consent was obtained form all participants. Animal experiments were reviewed and approved by the Ethics Committee on Animal Research of the Anhui Medical University (No. LLSC20180106).

Consent for publication

Not applicable.

Availability of data and materials

All materials and data used in the study are available and can be provided as necessary by contacting the corresponding author.

Competing interests

The authors declare that they have no competing interests.

\section{Funding}

This work is supported by National Natural Science Foundation of China (no. 81801511 and no.81802586). The funders had no role in the design of the study, in the collection, analysis, and interpretation of the data, or in the writing or approval of the manuscript.

\section{Authors' contributions}

SS proposed and supervised the project, designed the study and analyzed data. ZF performed experiments under the supervision of SS and BW. YH and CS helped with sample collection and western blotting. LZ, GZ helped with analysis and interpretation of data. SS, ZF and YG wrote the manuscript. All authors read and approved the final manuscript. 
The authors express their sincere thanks to all the participants in this study and Wenyan Wang, Yu Zhang, Enxue Song and Yijun Fan for their technical assistance.

\section{References}

1. Healy MW, Schexnayder B, Connell MT, Terry N, DeCherney AH, Csokmay JM, et al. Intrauterine adhesion prevention after hysteroscopy: a systematic review and meta-analysis. Am J Obstet Gynecol. 2016;215:267-75 e7. https://doi.org/10.1016/j.ajog.2016.05.001.

2. Hooker AB, Lemmers M, Thurkow AL, Heymans MW, Opmeer BC, Brolmann HA, et al. Systematic review and meta-analysis of intrauterine adhesions after miscarriage: prevalence, risk factors and long-term reproductive outcome. Hum Reprod Update. 2014;20:262-78. https://doi.org/10.1093/humupd/dmt045.

3. March CM. Asherman's syndrome. Semin Reprod Med. 2011;29:83-94. https://doi.org/10.1055/s0031-1272470.

4. Zhao G, Cao Y, Zhu X, Tang X, Ding L, Sun H, et al. Transplantation of collagen scaffold with autologous bone marrow mononuclear cells promotes functional endometrium reconstruction via downregulating DeltaNp63 expression in Asherman's syndrome. Sci China Life Sci. 2017;60:404-16. https://doi.org/10.1007/s11427-016-0328-y.

5. Xiao L, Song Y, Huang W, Yang S, Fu J, Feng X, et al. Expression of SOX2, NANOG and OCT4 in a mouse model of lipopolysaccharide-induced acute uterine injury and intrauterine adhesions. Reprod Biol Endocrinol. 2017;15:14. https://doi.org/10.1186/s12958-017-0234-9.

6. Sahin Ersoy G, Zolbin MM, Cosar E, Moridi I, Mamillapalli R, Taylor HS. CXCL12 Promotes Stem Cell Recruitment and Uterine Repair after Injury in Asherman's Syndrome. Mol Ther Methods Clin Dev. 2017;4:169-77. https://doi.org/10.1016/j.omtm.2017.01.001.

7. Liu D, Ha C, Zhang X, Zhang Z, Liu P. Molecular implication of ADAM-15 and -17 in intrauterine adhesions. Eur J Obstet Gynecol Reprod Biol. 2013;170:264-9.

https://doi.org/10.1016/j.ejogrb.2013.06.036.

8. Chen L, Zhang H, Wang Q, Xie F, Gao S, Song Y, et al. Reproductive Outcomes in Patients With Intrauterine Adhesions Following Hysteroscopic Adhesiolysis: Experience From the Largest Women's Hospital in China. J Minim Invasive Gynecol. 2017;24:299-304.

https://doi.org/10.1016/j.jmig.2016.10.018.

9. Gan L, Duan H, Xu Q, Tang YQ, Li JJ, Sun FQ, et al. Human amniotic mesenchymal stromal cell transplantation improves endometrial regeneration in rodent models of intrauterine adhesions. Cytotherapy. 2017;19:603-16. https://doi.org/10.1016/j.jcyt.2017.02.003.

10. Santamaria X, Cabanillas S, Cervello I, Arbona C, Raga F, Ferro J, et al. Autologous cell therapy with CD133+ bone marrow-derived stem cells for refractory Asherman's syndrome and endometrial atrophy: a pilot cohort study. Hum Reprod. 2016;31:1087-96. https://doi.org/10.1093/humrep/dew042. 
11. Chen Y, Liu L, Luo Y, Chen M, Huan Y, Fang R. Effects of Aspirin and Intrauterine Balloon on Endometrial Repair and Reproductive Prognosis in Patients with Severe Intrauterine Adhesion: A Prospective Cohort Study. Biomed Res Int. 2017;2017:8526104. https://doi.org/10.1155/2017/8526104.

12. Hu J, Zeng B, Jiang $X$, Hu L, Meng Y, Zhu Y, et al. The expression of marker for endometrial stem cell and fibrosis was increased in intrauterine adhesious. Int J Clin Exp Pathol. 2015;8:1525-34.

13. Deane JA, Gualano RC, Gargett CE. Regenerating endometrium from stem/progenitor cells: is it abnormal in endometriosis, Asherman's syndrome and infertility? Curr Opin Obstet Gynecol. 2013;25:193-200. https://doi.org/10.1097/GC0.0b013e32836024e7.

14. Wang J, Ju B, Pan C, Gu Y, Zhang Y, Sun L, et al. Application of Bone Marrow-Derived Mesenchymal Stem Cells in the Treatment of Intrauterine Adhesions in Rats. Cell Physiol Biochem. 2016;39:155360. https://doi.org/10.1159/000447857.

15. Lucas ES, Dyer NP, Fishwick K, Ott S, Brosens JJ. Success after failure: the role of endometrial stem cells in recurrent miscarriage. Reproduction. 2016;152:R159-66. https://doi.org/10.1530/REP-160306.

16. Gargett CE, Schwab KE, Deane JA. Endometrial stem/progenitor cells: the first 10 years. Hum Reprod Update. 2016;22:137-63. https://doi.org/10.1093/humupd/dmv051.

17. Chang MS, McNinch J, Basu R, Simonet S. Cloning and characterization of the human neutrophilactivating peptide (ENA-78) gene. J Biol Chem. 1994;269:25277-82.

18. Feng Z, Zhou H, Ma S, Guan X, Chen L, Huang J, et al. FTY720 attenuates intestinal injury and suppresses inflammation in experimental necrotizing enterocolitis via modulating CXCL5/CXCR2 axis. Biochem Biophys Res Commun. 2018;505:1032-7. https://doi.org/10.1016/j.bbrc.2018.10.013.

19. Konduru AS, Lee BC, Li JD. Curcumin suppresses NTHi-induced CXCL5 expression via inhibition of positive IKKbeta pathway and up-regulation of negative MKP-1 pathway. Sci Rep. 2016;6:31695. https://doi.org/10.1038/srep31695.

20. Wang C, Li A, Yang S, Qiao R, Zhu X, Zhang J. CXCL5 promotes mitomycin C resistance in nonmuscle invasive bladder cancer by activating EMT and NF-kappaB pathway. Biochem Biophys Res Commun. 2018;498:862-8. https://doi.org/10.1016/j.bbrc.2018.03.071.

21. Wang X, Ma N, Sun Q, Huang C, Liu Y, Luo X. Elevated NF-kappaB signaling in Asherman syndrome patients and animal models. Oncotarget. 2017;8:15399-406. https://doi.org/10.18632/oncotarget.14853.

22. Xue X, Chen Q, Zhao G, Zhao JY, Duan Z, Zheng PS. The Overexpression of TGF-beta and CCN2 in Intrauterine Adhesions Involves the NF-kappaB Signaling Pathway. PLoS One. 2015;10:e0146159. https://doi.org/10.1371/journal.pone.0146159.

23. Dang H, Wu W, Wang B, Cui C, Niu J, Chen J, et al. CXCL5 Plays a Promoting Role in Osteosarcoma Cell Migration and Invasion in Autocrine- and Paracrine-Dependent Manners. Oncol Res. 2017;25:177-86. https://doi.org/10.3727/096504016X14732772150343. 
24. Gao Y, Guan Z, Chen J, Xie H, Yang Z, Fan J, et al. CXCL5/CXCR2 axis promotes bladder cancer cell migration and invasion by activating PI3K/AKT-induced upregulation of MMP2/MMP9. Int J Oncol. 2015;47:690-700. https://doi.org/10.3892/ijo.2015.3041.

25. Nielepkowicz-Gozdzinska A, Fendler W, Robak E, Kulczycka-Siennicka L, Gorski P, Pietras T, et al. The Role of CXC Chemokines in Pulmonary Fibrosis of Systemic Lupus Erythematosus Patients. Arch Immunol Ther Exp (Warsz). 2015;63:465-73. https://doi.org/10.1007/s00005-015-0356-8.

26. Abe I, Teshima Y, Kondo H, Kaku H, Kira S, Ikebe Y, et al. Association of fibrotic remodeling and cytokines/chemokines content in epicardial adipose tissue with atrial myocardial fibrosis in patients with atrial fibrillation. Heart Rhythm. 2018. https://doi.org/10.1016/j.hrthm.2018.06.025.

27. Hu S, Li Y, Meng WJ, Tan SQ. [Effects of Fukang oral liquid on the prevention of intrauterine adhesion and expressions of TGF-beta1, PAI-1 and MMP-9 in endometrium of rats]. Sichuan Da Xue Xue Bao Yi Xue Ban. 2013;44:540-4.

28. Sun L, Zhang S, Chang Q, Tan J. Establishment and comparison of different intrauterine adhesion modelling procedures in rats. Reprod Fertil Dev. 2019. https://doi.org/10.1071/RD18397.

29. Jiang ZM, Luo TX, Zheng HG, Chen XH, Kuan JQ. [Ultrastructural study of fallopian tubes after occlusive sterilization with phenol-mucilage]. Shengzhi Yu Biyun. 1991;11:35-8.

30. Kong D, Zhang L, Xu X, Zhang J, Li Y, Huang X. Small Intestine Submucosa Is a Potential Material for Intrauterine Adhesions Treatment in a Rat Model. Gynecol Obstet Invest. 2018;83:499-507. https://doi.org/10.1159/000479086.

31. Askari VR, Rahimi VB, Zamani P, Fereydouni N, Rahmanian-Devin P, Sahebkar AH, et al. Evaluation of the effects of Iranian propolis on the severity of post operational-induced peritoneal adhesion in rats. Biomed Pharmacother. 2018;99:346-53. https://doi.org/10.1016/j.biopha.2018.01.068.

32. Zhang X, Li J, Xie B, Wu B, Lei S, Yao Y, et al. Comparative Metabolomics Analysis of Cervicitis in Human Patients and a Phenol Mucilage-Induced Rat Model Using Liquid Chromatography Tandem Mass Spectrometry. Front Pharmacol. 2018;9:282. https://doi.org/10.3389/fphar.2018.00282.

33. Ma Y, Luo Y, Song L, Qin F, Hou L. [Pharmaceutical screening of the effective fraction from Smilax for treatment of chronic pelvic inflammatory disease]. Nan Fang Yi Ke Da Xue Xue Bao. 2013;33:145-9.

34. Shogan BD, Belogortseva N, Luong PM, Zaborin A, Lax S, Bethel C, et al. Collagen degradation and MMP9 activation by Enterococcus faecalis contribute to intestinal anastomotic leak. Sci Transl Med. 2015;7:286ra68. https://doi.org/10.1126/scitranslmed.3010658.

35. Roca H, Jones JD, Purica MC, Weidner S, Koh AJ, Kuo R, et al. Apoptosis-induced CXCL5 accelerates inflammation and growth of prostate tumor metastases in bone. J Clin Invest. 2018;128:248-66. https://doi.org/10.1172/JCI92466.

36. Wang L, Xu Z, Chen B, He W, Hu J, Zhang L, et al. The Role of Vascular Endothelial Growth Factor in Small-airway Remodelling in a Rat Model of Chronic Obstructive Pulmonary Disease. Sci Rep. 2017;7:41202. https://doi.org/10.1038/srep41202.

37. Sun S, Zhong X, Wang C, Sun H, Wang S, Zhou T, et al. BAP18 coactivates androgen receptor action and promotes prostate cancer progression. Nucleic Acids Res. 2016;44:8112-28. 


\section{Figures}

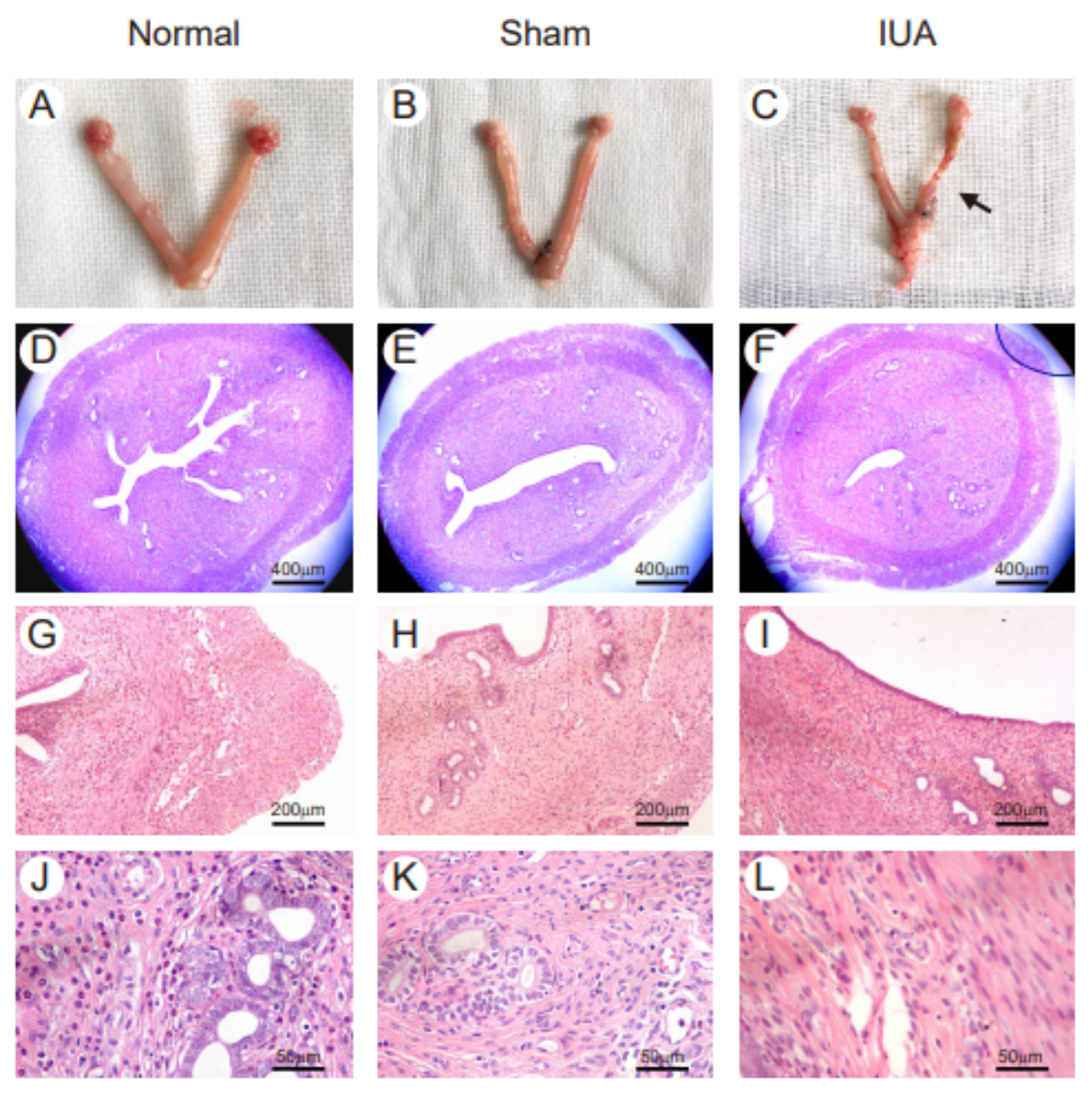

\section{Figure 1}

Abnormal morphology in IUA rats. (A-C) Representative images of the isolated uteruses from three groups of rats. Black arrowhead points to the intrauterine adhesion. (D-L) 40 rats were randomly separated into three different groups: normal group (10 rats with no treatment), sham-operated group (10 rats with vehicle treatment), IUA group (20 rats with phenol mucilage treatment). One side of the uterus in each rat was treated with phenol mucilage or vehicle treatment. The uteruses from three groups were collected for HE staining 14 days after treatment. Representative photomicrographs of uteruses sections obtained from three group rats at $40 \times(\mathrm{D}-\mathrm{F}$, scale bars: $400 \mu \mathrm{m}), 100 \times(\mathrm{G}-\mathrm{I}$, scale bars: $200 \mu \mathrm{m})$ and $400 \times(\mathrm{J}-\mathrm{L}$, scale bars: $50 \mu \mathrm{m}$ ) original magnification. In IUA rats, the uterine cavity was smaller than the other two groups. 


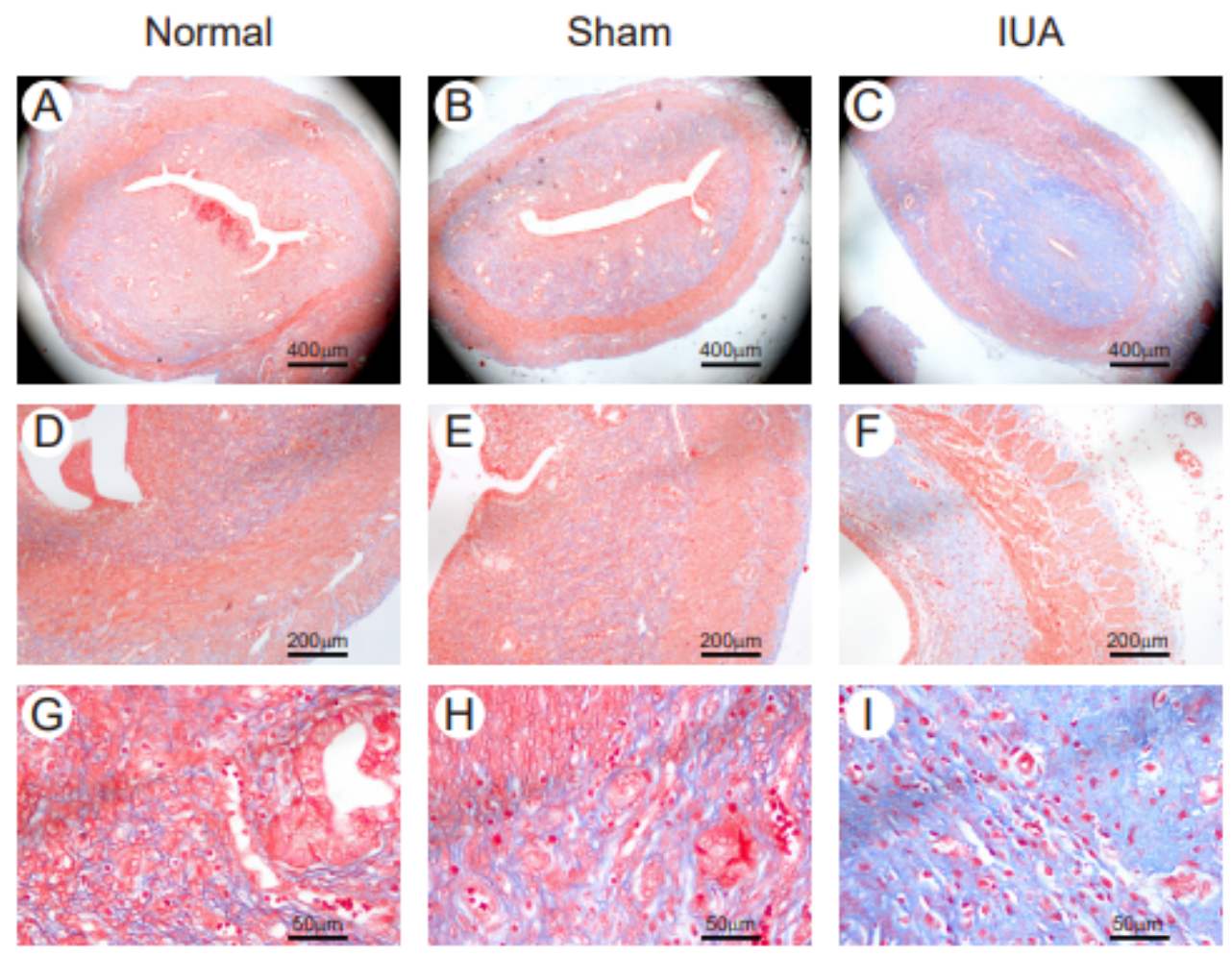

Figure 2

Masson staining for uteruses in three group rats. Selected representative images of uteruses in each group reveals the interstitial fibrosis changes. Blue staining in IUA rats demonstrated severer adhesion compared to normal and sham-operated rats. Normal group, $n=10$, Sham-operated group, $n=10$, IUA

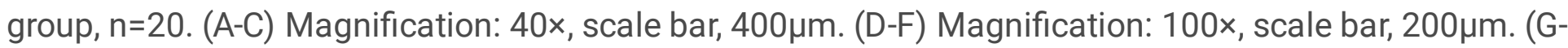
l) Magnification: $400 \times$, scale bar, $50 \mu \mathrm{m}$. 

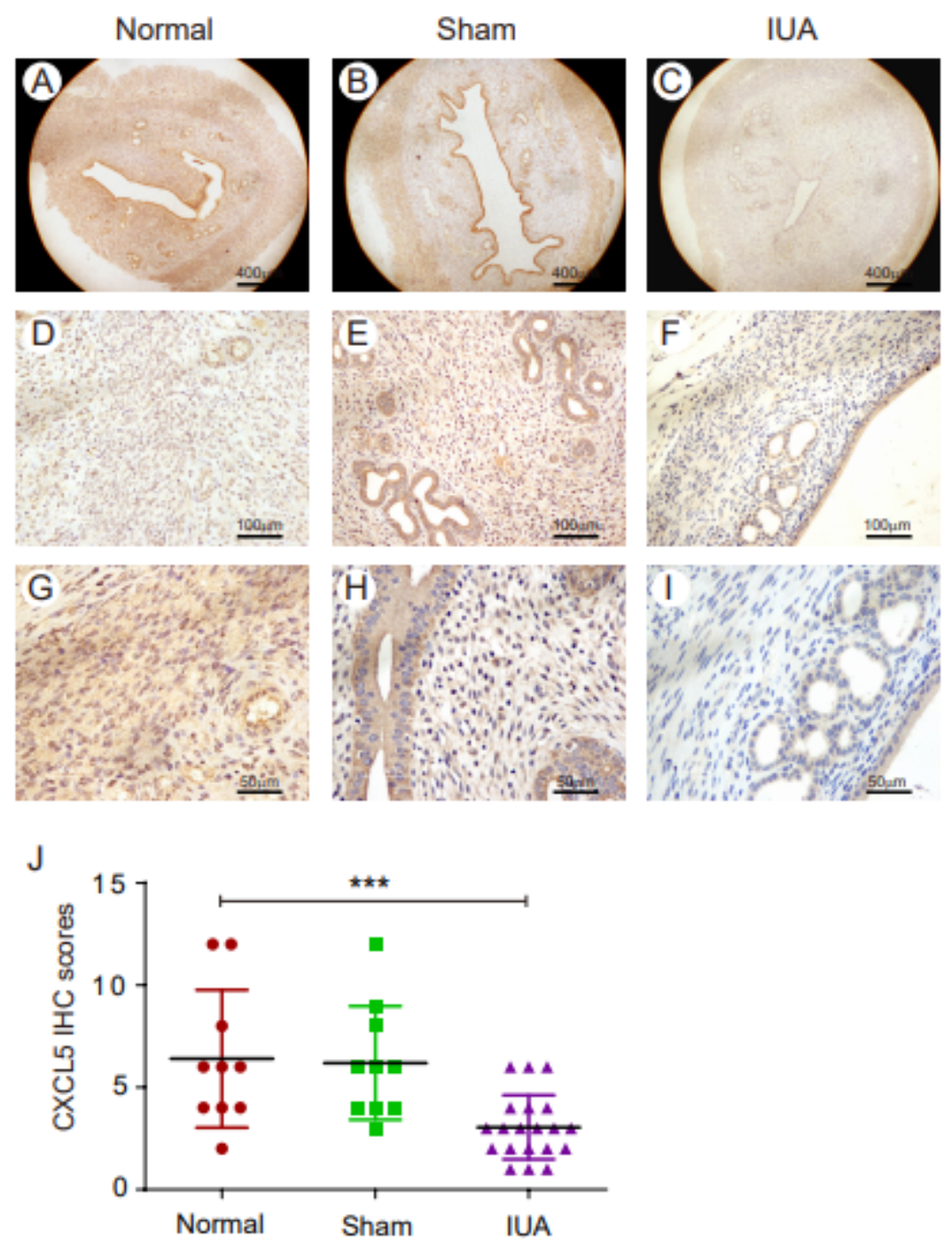

Figure 3

The expression of CXCL5 in IUA rats detected by IHC. (A-I) The representative localization of CXCL5 (brown stained) was observed in three different rat groups under light microscopy. Normal group, $n=10$, Sham-operated group, $n=10$, IUA group, $n=20$. (A-C) Magnification is $40 x$, and the scale bar is $400 \mu \mathrm{m}$. (DF) Magnification is $200 x$, and the scale bar is $100 \mu \mathrm{m}$. (G-I) Magnification is $400 x$, and the scale bar is $50 \mu \mathrm{m}$. (J) Summarization of proportion and intensity of positive staining. CXCL5 has lower staining in the IUA group compared with that in normal and sham-operated groups. Statistical significance of three groups was assessed, $* \star * P<0.001$. 
A

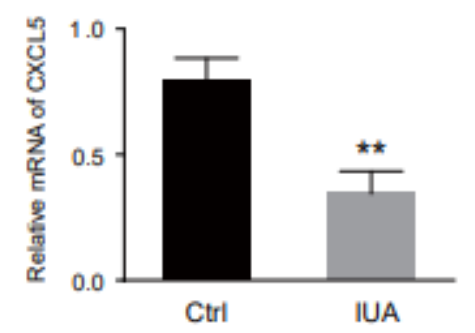

C

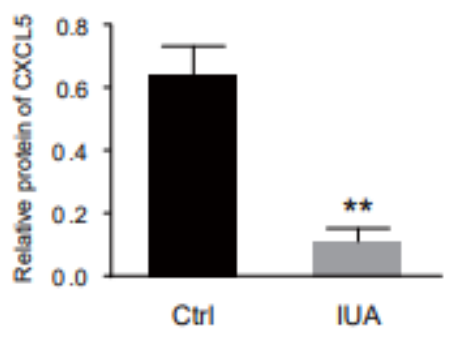

B

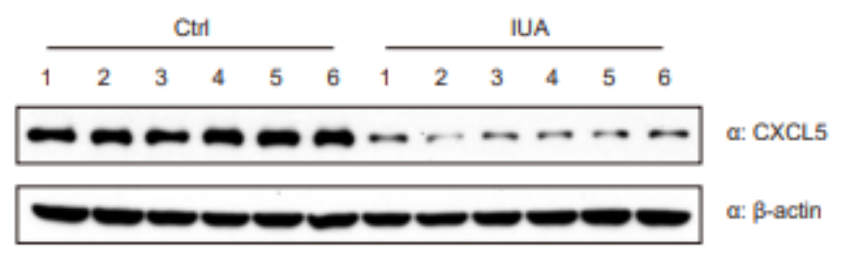

D

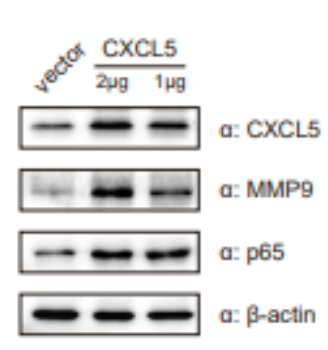

E

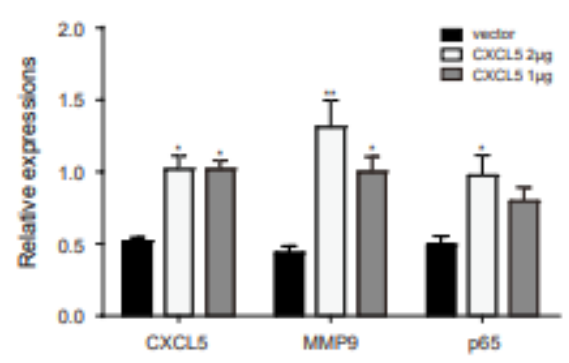

\section{Figure 4}

Down-expression of CXCL5 in IUA endometrium and the correlation between CXCL5, MMP9, and p65. (A) The expression of CXCL5 in 6 cases of normal endometria (Ctrl), 6 cases of IUA were measured by realtime PCR. Levels of all mRNA were normalized to that of GAPDH mRNA. (B) The protein levels of CXCL5 in endometrial tissues of two groups were detected by Western blotting. (C) A quantitative analysis of CXCL5 expression in IUA and normal endometrium tissues normalized to $\beta$-actin expression. (D) Ishikawa cells were transfected with CXCL5 $(2 \mu \mathrm{g}, 1 \mu \mathrm{g})$ or vector, and the expression of CXCL5, MMP9, and p65 was measured by western blotting. $\beta$-actin was used as an internal control. (E) Quantitative analysis of relative protein expression is shown. Statistical significance of differences between the two groups was assessed t-test. ${ }^{*} \mathrm{P}<0.05,{ }^{*} \mathrm{P}<0.01$.

\section{Supplementary Files}

This is a list of supplementary files associated with this preprint. Click to download.

- OriginallmagesofWB.pdf

- ARRIVEGuidelinesChecklistCXCL5inIUA.docx 